\title{
Predictive Factors of Late-onset Rectal Mucosal Changes After Radiotherapy of Prostate Cancer
}

\author{
EDY IPPOLITO ${ }^{1}$, ALESSANDRA GUIDO ${ }^{2}$, GABRIELLA MACCHIA $^{3}$, FRANCESCO DEODATO ${ }^{3}$, \\ LUCIA GIACCHERINI ${ }^{2}$, ANDREA FARIOLI ${ }^{4}$, ALESSANDRA ARCELLI ${ }^{2}$, DAJANA CUICCHI $^{4}$, \\ LEONARDO FRAZZONI ${ }^{4}$, SAVINO CILLA ${ }^{5}$, MILLY BUWENGE ${ }^{2}$, GIOVANNA MANTINI ${ }^{6}$, \\ ANNA R. ALITTO $^{6}$, MARIANNA NUZZO ${ }^{3}$, VINCENZO VALENTINI ${ }^{6}$, \\ MARCELLO INGROSSO ${ }^{7}$, ALESSIO G. MORGANTI ${ }^{2 *}$ and LORENZO FUCCIO ${ }^{4 *}$ \\ ${ }^{1}$ Radiotherapy Unit, Campus Bio-Medico University, Rome, Italy; \\ ${ }^{2}$ Radiation Oncology Center, Department of Experimental, \\ Diagnostic and Specialty Medicine DIMES, University of Bologna, Bologna, Italy; \\ ${ }^{3}$ Radiotherapy Unit, ${ }^{5}$ Medical Physics Unit, ${ }^{7}$ Endoscopy Unit, \\ Giovanni Paolo II Research and Care Foundation, Campobasso, Italy; \\ ${ }^{4}$ Department of Medical and Surgical Sciences (DIMEC), University of Bologna, Bologna, Italy; \\ ${ }^{6}$ Department of Radiotherapy, A. Gemelli Hospital, Sacred Heart Catholic University, Rome, Italy
}

\begin{abstract}
Background/Aim: The Vienna Rectoscopy Score (VRS; from 0, absence of rectal mucosal changes, to 5) assessed 1 year after radiotherapy is a surrogate end-point of late rectal toxicity. The aim of this study was to investigate the association between treatment-related factors and 1-year VRS. Patients and Methods: We performed a retrospective analysis of prospectively collected data. Patients with prostate adenocarcinoma treated with definitive or postoperative radiotherapy $(R T)$ underwent endoscopy 1 year after RT. Relationships between VRS of 2 or more and treatment parameters were investigated by univariate and multivariate logistic analyses. Results: One hundred and ninety-five patients ( mean age $=69$ years; range $=43-81$ years) were considered eligible for the study. At univariate analysis, patients treated with hypofractionation plus radiosurgery boost $(p<0.001)$ and an equivalent dose in 2 Gy per fraction (EQD2) $(\alpha / \beta=3) \geq 75$ Gy $(p<0.001)$ was associated with a significantly higher incidence of $V R S \geq 2$ after 1 year of
\end{abstract}

This article is freely accessible online.

*These Authors contributed equally to this study.

Correspondence to: Alessandra Guido, MD, Ph.D., Radiation Oncology Center, Department of Experimental, Diagnostic and Specialty Medicine, University of Bologna, Via Massarenti 9, 40138 Bologna, Italy. E-mail: alessandraguido2008@gmail.com

Key Words: Rectal toxicity, prostate cancer radiotherapy, radiationinduced GI toxicity. follow-up. At multivariate analysis, radiosurgery boost was an independent risk factor for developing rectal mucosal lesions (VRS $\geq 2$ ), yielding an odds ratio (OR) of $4.14(95 \%$ confidence interval $(C I)=1.2-13.8)$, while pelvic surgery was inversely associated with $V R S \geq 2(O R=0.39$; $95 \%$ CI=0.170.94). Conclusion: Hypofractionation followed by radiosurgery boost significantly increased the risk of developing late-onset rectal mucosal changes. Therefore, special care and preventative treatment strategies are needed when using radiosurgery boost after hypofractionated $R T$.

Rectal late injury represents a major concern in prostate cancer radiotherapy (RT), including several symptoms such as rectal bleeding, urgency, frequency and loose stool (1-3). These complications become greater when higher doses are used. Recently, newer techniques such as intensitymodulated (IMRT), volumetric arc therapy (VMAT) and image-guided RT allow the delivery of higher doses while promoting rectal sparing (4-6). However, despite technological improvements, the incidence rate of late grade 2 proctopathy is reduced but not abolished, ranging between $3 \%$ and $25 \%(7,8)$.

Rectosigmoidoscopy can provide an early and accurate estimation of radiation-induced rectal mucosal damage by identifying pre-existing pathological conditions and detecting signs of tissue changes below the level of subjective symptoms (9). Watcher et al. (10) first used the Vienna Rectoscopy Score (VRS) based on the endoscopic terminology of the World Organization for Digestive Endoscopy (OMED), as published by the European Society for Gastrointestinal 
Endoscopy (11) to describe rectal mucosal changes after pelvic RT. VRS was shown to be cosistent with clinical symptoms assessed by different toxicity scales $(12,13)$.

It has been shown that VRS on rectosigmoidoscopy performed 1 year after the end of RT can predict late-onset rectal toxicity and can, therefore, be used as a surrogate endpoint (14).

Regarding the role of clinical factors, such as hemorrhoids, antihypertensive and anticoagulant drugs, diabetes, prior pelvic surgery and inflammatory bowel symptoms, in increasing late rectal toxicity, conflicting results have been reported. On the other hand, there is more agreement in recognizing the role of clinical factors as predictive parameters of late rectal toxicity (15).

Based on the above considerations, the aim of this study was to investigate the correlation between treatment parameters and VRS score evaluated by rectosigmoidoscopy 1 year after the end of radiation treatment.

\section{Patients and Methods}

Study design. This study represents a retrospective analysis of a prospectively collected database. The database of the Giovanni Paolo II Research and Care Foundation in Campobasso (Italy) was reviewed. The study was approved by the Ethical Committee (201407-053) and written informed consent for the procedure and data acquisition was obtained from all patients.

Study population. All patients affected by prostate adenocarcinoma and treated with RT in definitive or postoperative setting who underwent a lower endoscopy examination 1 year after the end of radiation treatment were included in the analysis.

Radiotherapy. Patients underwent 3D conformal technique or IMRT or VMAT according to different radiation protocols. A 5-Gy radiosurgery boost was delivered to patients in the definitive treatment group (intermediate-high risk) after moderate hypofrationated radiotherapy (65 Gy/2.6 Gy per fraction) with VMAT technique. Radiosurgery boost was delivered in order to increase the biologically equivalent dose while reducing the overall treatment time. Clinical target volume 1 (CTV1) included the prostate only or the prostate and seminal vesicles in the definitive treatment group and prostate bed in the postoperative group. CTV2 was defined as CTV1 plus pelvic lymph nodes (presacral, obturator, internal iliac and external iliac chains). CTV for radiosurgery boost included the whole prostate with or without disease extension to seminal vesicles. Only patients presenting a $>15 \%$ risk of nodal involvement according to the Roach formula (16) received pelvic nodal irradiation. Planning target volume 1 (PTV1) and PTV2 were defined by adding an $8 \mathrm{~mm}$ margin (6 mm posteriorly) to the CTV1 and CTV2, respectively. Before simulation and treatment, patients were asked to spontaneously empy the rectum or otherwise to undergo an enema. Daily portal images were used for set-up verification. Implanted fiducial markers were employed to account for organ motion.

Endoscopy. The endoscopic assessment was performed with flexible sigmoidoscopy. All the procedures were performed by trained endoscopists (with at least 5 years experience and more than 250
Table I. Patient characteristics.

\begin{tabular}{lcc}
\hline Characteristic & $\mathrm{N}$ & $\%$ \\
\hline Total patients & 195 & 100.0 \\
Radiotherapy & 152 & 77.9 \\
Definitve & 43 & 22.1 \\
Postoperative or salvage & & \\
Total EQD2 dose (Gy) & 126 & 64.6 \\
$\quad$ <75 Gy & 69 & 35.4 \\
$\quad \geq 75$ Gy & & \\
Seminal vesicle irradiation & 181 & 92.8 \\
$\quad$ Yes & 14 & 7.2 \\
$\quad$ No & 169 & 86.7 \\
Prophylactic nodal irradiation & 26 & 13.3 \\
$\quad$ Yes & & \\
$\quad$ No & 65 & 33.3 \\
Radiation technique & 130 & 66.7 \\
3-D & & \\
IMRT/VMAT & 59 & 30.3 \\
Hypofractionation with radiosurgery boost & 136 & 69.7 \\
$\quad$ Yes & & \\
$\quad$ No & 39 & 21.6 \\
Hypofractionation without radiosurgery boost & 141 & 78.3 \\
$\quad$ Yes & & \\
No & 183 & 93.8 \\
Androgen deprivation therapy & 12 & 6.2 \\
$\quad$ Yes & & \\
No & & \\
\hline
\end{tabular}

IMRT/VMAT: Intensity-modulated/volumetric arc therapy; EQD2: equivalent dose delivered in 2 Gy fractions.

procedures per year). Endoscopic findings were described using the World Organisation of Digestive Endoscopy terminology (11) and evaluated according to the VRS (10). Briefly, the VRS considered presence and severity of five parameters: mucosal congestion, telangiectasia, ulcer, stricture and necrosis. The VRS ranged from 0 (absence of rectal mucosal changes) to 5. Baseline endoscopic evaluation was not performed.

Statistical analysis. For each patient, the following clinical data were recorded: previous pelvic surgery, radiotherapy technique, androgen deprivation therapy, total equivalent dose calculated with the equivalent dose in 2 Gy fraction (EQD2) equation using an $\alpha / \beta$ value of 3 , seminal vesicle irradiation, prophylactic nodal irradiation, radiosurgery boost and hypofractionation. We used an $\alpha / \beta$ value of 3 as the primary endpoint of the study to evaluate late rectal changes described by VRS.

The investigated endpoint was rectal mucosal changes detected by endoscopy, scored with VRS, and dichotomized as follows: VRS: $<2$ versus $\mathrm{VRS} \geq 2$. We chose this cut-off since the frequency of $\mathrm{VRS} \geq 2$ was greater in the study population. Differences in clinical data between patients presenting a VRS score $<2$ or $\geq 2$ at endoscopy were tested by Pearson chi-square test. In order to assess the relation between clinical data and the risk of developing a VRS score $\geq 2$, odds ratios (ORs) were computed by logistic regression at univariate and multivariate analyses. The statistical analysis was performed using SYSTAT, version 12.0 (SPSS, Chicago, IL, USA). 
Table II. Endoscopy findings

\begin{tabular}{lcc}
\hline VRS & No. of patients & $\%$ \\
\hline 0 & 14 & $7.2 \%$ \\
1 & 41 & $21.0 \%$ \\
2 & 70 & $35.9 \%$ \\
3 & 61 & $31.3 \%$ \\
4 & 7 & $3.6 \%$ \\
5 & 2 & $1.0 \%$ \\
\hline
\end{tabular}

VRS: Vienna Rectoscopy Score.

\section{Results}

Study population. One hundred and ninety-five patients (mean age: 69; range: 43-81) were considered eligible in the study: 152 patients $(77.9 \%)$ were treated with curative RT and 169 patients $(86.7 \%)$ received prophylactic nodal irradiation. The median EQD2Gy $(\alpha / \beta=3)$ was $70.20 \mathrm{~Gy}$ (range=59.81-73.00 Gy) and 75 Gy (range=70.00- 80.80 Gy) for the postoperative and definitive group respectively. Hypofractionation was used in 98 patients (50.3\%). Patient characteristics are reported in Table I.

Endoscopic findings. One hundred and twenty-five patients $(64.1 \%)$ presented a VRS score $\geq 2$ and 70 patients $(35.9 \%)$ a VRS score $\geq 3$. Rectal ulceration was reported in two cases $(1 \%)$. Fourteen patients $(7.2 \%)$ did not show any mucosal alterations. Detailed results are given in Table II.

$V R S$ and clinical variables. At univariate analysis, patients treated with an EQD2 dose $\geq 75$ Gy $(p<0.001)$, with hypofractionation plus radiosurgery boost $(p<0.001)$, with seminal vesicles irradiation $(p=0.013)$, had a higher incidence of VRS $\geq 2$, while previous surgery ( $p<0.001)$, and $3 \mathrm{D}$-conformal radiotherapy $(p=0.003)$ were associated with a lower incidence of rectal mucosal changes. All the results of the Pearson Chi-square test comparing clinical variables between patients with and without rectal mucosal changes are shown in Table III.

At multivariate analysis, radiosurgery boost $(p=0.02)$ and prior pelvic surgery $(p=0.03)$ were significantly associated with the risk of developing a VRS $\geq 2$. Indeed, patients treated with a radiosurgery boost presented an increased risk $(\mathrm{OR}=4.14(95 \% \mathrm{CI}=1.24-13.81)$, while patients surgically treated presented a reduced risk $(\mathrm{OR}=0.39(95 \% \mathrm{CI}=0.17$ $0.94)$. Radiosurgery boost and EQD2 $>75$ Gy were strongly correlated (Spearman's rank correlation $=0.890, p=<0.001$ ), consequently we only included radiosurgery boost in the multivariate analysis. The results of multivariate logistic regression are also shown in Table IV.
Table III. Results of Pearson chi-square test assessing differences for treatment data between the patients with a Vienna Rectoscopy Score score $<2$ and $\geq 2$.

\begin{tabular}{|c|c|c|c|}
\hline \multirow[b]{2}{*}{ Dosimetric parameter } & \multicolumn{3}{|c|}{ Vienna Rectoscopy Score } \\
\hline & $<2$ & $\geq 2$ & $p$-Value \\
\hline \multicolumn{4}{|l|}{ Surgery $(\%)$} \\
\hline Yes & 51.2 & 48.8 & \multirow[t]{2}{*}{$<0.001$} \\
\hline No & 21.7 & 78.3 & \\
\hline \multicolumn{4}{|l|}{ RT technique (\%) } \\
\hline 3D-CRT & 41.5 & 58.5 & \multirow[t]{2}{*}{0.003} \\
\hline IMRT/VMAT & 21.5 & 78.5 & \\
\hline \multicolumn{4}{|c|}{ Androgen deprivation therapy $(\%)$} \\
\hline Yes & 28.4 & 71.6 & \multirow[t]{2}{*}{0.799} \\
\hline No & 25.0 & 75.0 & \\
\hline \multicolumn{4}{|l|}{ EQD2 dose $(\%)$} \\
\hline$<75$ Gy & 36.5 & 63.5 & \multirow[t]{2}{*}{$<0.001$} \\
\hline$>75$ Gy & 13.0 & 87.0 & \\
\hline \multicolumn{4}{|c|}{ Seminal vesicle irradiation (\%) } \\
\hline Yes & 26.0 & 74.0 & \multirow[t]{2}{*}{0.013} \\
\hline No & 57.1 & 42.9 & \\
\hline \multicolumn{4}{|c|}{ Prophylactic node irradiation (\%) } \\
\hline Yes & 26.6 & 73.4 & \multirow[t]{2}{*}{0.212} \\
\hline No & 38.5 & 61.5 & \\
\hline \multicolumn{4}{|l|}{$\begin{array}{l}\text { Hypofractionation with } \\
\text { radiosurgery boost (\%) }\end{array}$} \\
\hline Yes & 6.8 & 93.2 & \multirow[t]{2}{*}{$<0.001$} \\
\hline No & 37.5 & 62.5 & \\
\hline \multicolumn{4}{|c|}{$\begin{array}{l}\text { Hypofractionation without } \\
\text { radiosurgery boost (\%) }\end{array}$} \\
\hline Yes & 25.0 & 53.3 & \multirow[t]{2}{*}{0.286} \\
\hline No & 5.0 & 16.7 & \\
\hline
\end{tabular}

IMRT/VMAT: Intensity-modulated/volumetric arc therapy; EQD2: equivalent dose delivered in $2 \mathrm{~Gy}$ fractions.

\section{Discussion}

This study shows that the risk of developing multiple rectal angiectasias (i.e. VRS $\geq 2$ ) is increased in patients treated with higher RT doses, while it seems to be reduced in those with a history of pelvic surgery.

Our findings, if confirmed by large, prospective, multicenter studies, would appear meaningful because it further increases our understanding of RT-induced gastrointestinal (GI) toxicity, which may help clinicians to identify patients at higher risk of toxicity and propose preventive treatment strategies. Interestingly, at multivariate analysis, the strongest predictor of VRS $\geq 2$ was radiosurgery boost ( $5 \mathrm{~Gy})(\mathrm{OR}=4.14 ; \mathrm{CI}=1.24-13.81 ; p=0.001)$ following moderately hypofractionated RT.

Recent studies evaluated the use of stereotactic RT boost to the prostate after standard fractionation pelvic RT in order to escalate biologically effective dose in intermediate- and high-risk prostate cancer (17-19). With a median follow-up greater than 42 months, limited grade 2 and no grade $3 \mathrm{GI}$ 
in vivo $31: 961-966(2017)$

Table IV. Univariate and multivariate logistic regression for Vienna Rectoscopy Score score $\geq 2$.

\begin{tabular}{|c|c|c|c|c|c|c|}
\hline \multirow[b]{2}{*}{ Variable } & \multicolumn{3}{|c|}{ Univariate } & \multicolumn{3}{|c|}{ Multivariate } \\
\hline & OR & $95 \% \mathrm{CI}$ & $p$-Value & OR & $95 \% \mathrm{CI}$ & $p$-Value \\
\hline Surgery & 0.265 & $0.130-0.539$ & $<0.001$ & 0.397 & $0.167-0.944$ & 0.037 \\
\hline IMRT/VMAT & 2.588 & $1.356-4.942$ & 0.004 & 2.097 & $0.960-4.581$ & 0.063 \\
\hline EQD2 dose $\geq 75$ Gy & 3.833 & $1.742-8.437$ & 0.001 & - & - & - \\
\hline Seminal vesicle irradiation & 3.801 & $1.254-11.528$ & 0.018 & 2.075 & $0.577-7.463$ & 0.264 \\
\hline Radiosurgery boost & 8.250 & $2.822-24.117$ & $<0.001$ & 4.143 & $1.243-13.812$ & 0.021 \\
\hline
\end{tabular}

IMRT/VMAT: Intensity-modulated/volumetric arc therapy; EQD2: equivalent dose delivered in 2 Gy fractions; OR: odds ratio; CI: confidence interval.

toxicities were recorded. Additionally, several studies experimented with extreme hypofractionation ( $>5$ Gy per fraction) for prostate cancer using high-dose brachytherapy and stereotactic body radiotherapy (SBRT) alone. Only phase II prospective studies with SBRT have been published, reporting a low-moderate rate of grade 2 or more GI toxicity in about $2-16 \%$ of cases, with a minimum biologically equivalent dose delivered of 85 Gy (20).

Our results differed from previous studies for several reasons (17-20). Firstly, we focused our attention on subclinical mucosal changes instead of clinical symptoms, which can have a different biological evolution over the time, since altered fractionation was used. Interestingly, in a recent retrospective study conducted by Sood et al. which described rectal endoscopic findings following SBRT (median time from SBRT to proctoscopy=13 months) either as primary treatment or boost, of the 10 patients (20\%) with late rectal bleeding only four had evidence of teleangectasia (21). Secondly, the group of patients who received radiosurgery boost included intermediate-high-risk patients who underwent RT to seminal vesicles, which is a recognized risk factor for increased GI toxicity. Thirdly, patients were treated after moderate hypofractionation. A recent phase III trial conducted in the Netherlands on late (3 years after RT for prostate cancer) GI toxicity for hypofractionation compared to standard fractionation (22) confirmed two previous randomized trials $(23,24)$. These previous phase III trials on moderate hypofractionation for prostate cancer showed that there was any significant difference between conventional fractionation and hypofractionation in term of late GI toxicity. Thus, the 5 Gy limited dose for our subset of patients treated with radiosurgery boost might be an additional factor increasing risk of rectal toxicity.

In our study, a history of previous pelvic surgery significantly reduced the risk of developing late rectal mucosal changes, yielding an OR of 0.39 (95\% CI $=0.17-$ 0.94). This is a controversial issue in the literature (25-27), however, we believe that in our series this finding was strongly influenced by the prescription dose which was lower in patients with prior pelvic surgery (median EQD2 $=70.2 \mathrm{vs}$. $75 ; p=0.01)$.

The identification of subgroup of patients with a higher risk of developing rectal toxicity has important clinical consequences. The pathogenesis of radiation-induced toxicity is a multistep process. The acute response, during the first weeks of radiation therapy, is an acute inflammatory reaction and the development of chronic toxicity is, at least in part, related to severity of this acute response, the so-called 'consequential effect' (28). However, inflammation has only a marginal role in the etiology of late-onset toxicity; vascular sclerosis, obliterative endoarterites, fibrosis and consequent neo-angiogenesis with predominant telangiectasias are the main processes that underlie chronic effects of RT. Thus, rectal bleeding is strictly related to this neo-angiogenic process, since these immature neo-vessels are particularly fragile and simple stool passage is sufficiently traumatic to induce bleeding. In order to prevent or reduce late-onset toxicity, several drugs have been tested with the intent of reducing the initial inflammatory response (29). In 2011, we reported our experience with the preventive treatment with beclomethasone dipropionate (BDP), a topical, non-systemic glucorticosteroid (30). The rationale of this approach is to reduce the damage to normal tissues that surround the irradiated fields by blocking the inflammatory process with BDP. One hundred and twenty patients were randomized to receive $\mathrm{BDP}$ or identical-looking placebo throughout the $\mathrm{RT}$ and for a further 4 weeks thereafter. After 12 months of follow-up, patients treated with BDP presented a significant reduction of post-radiation risk of bleeding $(\mathrm{OR}=0.38 ; 95 \%$ $\mathrm{CI}=0.17-0.86)$ and of rectal mucosal changes. In particular, actively treated patients presented fewer rectal angiectasias in comparison to non-treated patients. It could be argued that patients at higher risk of developing late-onset GI toxicity (e.g. patients treated with radiosurgery boost) might benefit from a BDP-based preventative treatment. 
In our study population, $\mathrm{VRS} \geq 2$ occurred mostly in patients who were irradiated to the seminal vesicles $(p=0.013)$ and who were treated with higher prescription dose (EQD2 $>75 \mathrm{~Gy} ; p<0.001)$. In these patients, larger volume of rectum usually receives higher radiation dose, thus increasing late rectal toxicity, especially rectal bleeding (25). Indeed, the volume of the rectum receiving 60 and $70 \mathrm{~Gy}$ as well as the mean dose delivered to the rectum are both predictors of VRS $\geq 2$ (31). Moreover, 3D-conformal RT was associated at univariate analysis, with a significantly lower incidence of rectal mucosal changes $(p=0.003)$. This finding was unexpected. The protective role of $3 \mathrm{D}$ RT compared to intensitymodulated therapies (IMRT/VMAT) may be misleading since it is well known that IMRT is expected to deliver a lower radiation dose to the rectum. However, we believe that this finding is mainly related to the lower prescription dose delivered with $3 \mathrm{D}$ compared to IMRT/VMAT (median EQD2 prescription dose $=72 \mathrm{vs} .75$ $\mathrm{Gy} ; p=0.02)$.

This study presents several drawbacks that should be acknowledged. Firstly, there might be bias due to the retrospective design of the study, even though it included all consecutive patients of a prospectively collected database. Secondly, the study population was heterogeneous. However, as each variable can individually affect the incidence of rectal mucosal changes, we preferred to include the whole population in the study. Thirdly, a baseline endoscopic assessment of rectal mucosa prior to RT was not performed, thus potentially leading to an underestimation of pre-existing mucosal alterations. However, several endoscopy-based studies have clearly shown that almost all patients have a VRS of 0 before the beginning of RT (30). We used the VRS as a surrogate endpoint of rectal toxicity since in our previous study it was shown to be strongly correlated with long-term rectal toxicity (31). More recently, in a large cohort of patients Fellin et al. showed that mild long-term rectal symptoms were strongly correlated with moderate/severe events occurring in the first 3 years after RT (32). This further supports the utility of an endoscopic examination performed at the end of RT.

In conclusion, our study showed that radiosurgery boost may significantly increase the risk of developing rectal mucosal changes and late toxicity, therefore special care and preventative treatment strategies are needed by using this boost technique after hypofractonated RT, even if low doses are used. Additionally, boosting to only the dominant intraprostatic tumor lesion may be a viable option, as some studies showed promising results (33). Further prospective multicentric studies are warranted to confirm our findings and allow for better understanding of the association between rectal mucosal angiectasias and treatment/patient factors.

\section{Conflicts of Interest}

No actual or potential conflicts of interest exist regarding this article.

\section{References}

1 Fuccio L, Guido A and Andreyev HJ: Management of intestinal complications in patients with pelvic radiation disease. Clin Gastroenterol Hepatol 10: 1326-1334, 2012.

2 Denham JW, O'Brien PC, Dunstan RH, Johansen J, See A, Hamilton CS, Bydder $\mathrm{S}$ and Wright $\mathrm{S}$ : Is there more than one late radiation proctitis syndrome? Radiother Oncol 51: 43-53, 1999.

3 Andreyev J: Gastrointestinal symptoms after pelvic radiotherapy: A new understanding to improve management of symptomatic patients. Lancet Oncol 8: 1007-1017, 2007.

4 Zelefsky MJ, Levin EJ, Hunt M, Yamada Y, Shippy AM, Jackson A and Amols HI: Incidence of late rectal and urinary toxicities after three-dimensional conformal radiotherapy and intensitymodulated radiotherapy for localized prostate cancer. Int J Radiat Oncol Biol Phys 70: 1124-1129, 2008.

5 Wolff D, Stieler F, Welzel G, Lorenz F, Abo-Madyan Y and Mai S, Herskind C, Polednik M, Steil V, Wenz F and Lohr F: Volumetric-modulated arc therapy (VMAT) vs. serial tomotherapy, step-and-shoot IMRT and 3D-conformal RT for treatment of prostate cancer. Radiother Oncol 93: 226-233, 2009.

6 Zhang P, Happersett L, Hunt M, Jackson A, Zelefsky M and Mageras G: Volumetric-modulated arc therapy: planning and evaluation for prostate cancer cases. Int J Radiat Oncol Biol Phys 76: 1456-1462, 2010.

7 Lock M, Best L, Wong E, Bauman G, D'Souza D, Venkatesan V, Sexton T, Ahmad B, Izawa J and Rodrigues G: A phase II trial of arc-based hypofractioned intensity-modulated radiotherapy in localized prostate cancer. Int J Radiat Oncol Biol Phys 80: 13061315, 2011.

8 Arcangeli G, Fowler J, Gomellini S, Arcangeli S, Saracino B, Petrongari MG, Benassi $M$ and Strigari L: Acute and late toxicity in a randomized trial of conventional versus hypofractioned three-dimensional conformal radiotherapy for prostate cancer. Int J Radiat Oncol Biol Phys 79: 1013-1021, 2011.

9 Andreyev HJ, Vlavianos P, Blake P,. Dearnaley D, Norman AR and Tait D: Gastrointestinal symptoms after pelvic radiotherapy: role for the gastroenterologist? Int J Radiat Oncol Biol Phys 62: 1464-1471, 2005.

10 Wachter S, Gerstner N, Goldner G, Potzi R, Wambersie A and Potter R: Endoscopic scoring of late rectal mucosal damage after conformal radiotherapy for prostatic carcinoma. Radiother Oncol 54: 11-19, 2000.

11 Crespi M, Delvaux M, Schaprio M, Venables C and Zwiebel F: Working party report by the committee for minimal standards of terminology and documentation in digestive endoscopy of the European Society of Gastrointestinal Endoscopy. Minimal standard terminology for a computerized endoscopic data base. Ad hoc task force of the committee. Am J Gastroenterol 91: 191216,1996.

12 Goldner G, Tomicek B, Becker G, P Geinitz H, Wachter S, Zimmermann F, Wachter-Gerstner N and Reibenwein J, Glocker S, Bamberg M, Feldmann H, Pötzi R, Molls M and Pötter R: 
Proctitis after external-beam radiotherapy for prostate cancer classified by Vienna Rectoscopy Score and correlated with EORTC/RTOG score for late rectal toxicity: results of a prospective multicenter study of 166 patients. Int J Radiat Oncol Biol Phys 67: 78-83, 2007.

13 Georg P, Kirisits C, Goldner G, Dörr W , Hammer J, Pötzi R, Berger D, Dimopoulos J, Georg D and Pötter R: Correlation of dose-volume parameters, endoscopic and clinical rectal side effects in cervix cancer patients treated with definitive radiotherapy including MRI-based brachytherapy. Radiother Oncol 91: 173-180, 2009.

14 Ippolito E, Massaccesi M, Digesù C, Deodato F, Macchia G, Pirozzi GA, Cilla S, Cuscunà D, Di Lallo A, Mattiucci GC, Mantini G, Pacelli F, Valentini V, Cellini N, Ingrosso M and Morganti AG: Early proctoscopy is a surrogate endpoint of late rectal toxicity in prostate cancer treated with radiotherapy. Int $\mathbf{J}$ Radiat Oncol Biol Phys 83: 191-195, 2012.

15 Fonteyne V, Ost P, Vanpachtenbeke F, Colman R, Sadeghi S, Villeirs G, Decaestecker K and De Meerleer G: Rectal toxicity after intensity-modulated radiotherapy for prostate cancer: Which rectal dose volume constraints should we use? Radiother Oncol 113: 398-403, 2014.

16 Roach M, Marquez C, You H, Narayan P, Coleman L, Nseyo UO, Navvab Z and Carroll PR: Predicting the risk of lymph node involvement using the pre-treatment prostate specific antigen and Gleason score in men with clinically localized prostate cancer. Int J Radiat Oncol Biol Phys 28: 33-37, 1994.

17 LinYW, Lin LC and Lin K: The early result of whole pelvic radiotherapy and stereotactic body radiotherapy boost for highrisk localized prostate cancer. Front Oncol 4: 278, 2014.

18 Katz A and Kang J: Stereotactic body radiotherapy with or without external beam radiation as treatment for organ confined high-risk prostate carcinoma: a six-year study. Radiat Oncol 9: 1, 2014.

19 Anwar M, Weinberg V, Seymour Z, Hsu IJ, Roach M 3rd and Gottschalk AR: Outcomes of hypofractionated stereotactic body radiotherapy boost for intermediate and high risk prostate cancer. Radiat Oncol 11: 8, 2016.

20 Koontz BF, Bossi A, Cozzarini C, Wiegel T and D'Amico A: A systematic review of hypofractionation for primary management of prostate cancer. Eur Urol 68: 683-691, 2015.

21 Sood S, Ju AW, Wang H, Lei S, Uhm S, Zhang G, Suy S, Carroll J, Lynch J, Dritschilo A and Collins SP: Rectal endoscopy findings following stereotactic body radiation therapy for clinically localized prostate cancer. Radiat Oncol 8: 197, 2013.

22 Aluwini S, Pos F, Schimmel E, Krol S, van der Toorn PP, de Jager H, Dirkx M, Alemayehu WG, Heemsbergen W, Heijmen $\mathrm{B}$ and Incrocci L: Hypofractionated versus conventionally fractionated radiotherapy for patients with prostate cancer (HYPRO): late toxicity results from a randomized, noninferiority, phase III trial. Lancet Oncol 17: 464-474, 2016.

23 Arcangeli G, Fowler J, Gomellini S, Arcangeli S, Saracino B, Petrongari MG, Benassi M and Strigari L: Acute and late toxicity in a randomized trial of conventional versus hypofractionated three-dimensional conformal radiotherapy for prostate cancer. Int J Radiat Oncol Biol Phys 79: 1013-1021, 2011.

24 Pollack A, Walker G, Horwitz EM, Price R, Feigenberg S, Konski AA, Stoyanova R, Movsas B, Greenberg RE, Uzzo RG, Ma C and Buyyounouski MK: Randomized trial of hypofractionated external-beam radiotherapy for prostate cancer. J Clin Oncol 31: 3860-3868, 2013.
25 Fellin G, Fiorino C, Rancati T, Vavassori V, Baccolini M, Bianchi C, Cagna E, Gabriele P, Mauro F, Menegotti L, Monti $\mathrm{AF}$, Stasi $\mathrm{M}$ and Valdagni R: Clinical and dosimetric predictors of late rectal toxicity after conformal radiation for localized prostate cancer: results of a large multi-center observational study. Radiother Oncol 93: 197-202, 2009.

26 Barnett GC, De Meerleer G, Gulliford SL, Sydes MR, Elliott RM and Dearnaley DP: The impact of clinical factors on the development of late radiation toxicity: results from the medical research council RT01 Trial (ISRCTN47772397). Clin Oncol 23: 613-624, 2011.

27 Valdagni R, Vavassori V, Rancati T, Fellin G, Baccolini M, Bianchi C, Cagna E, Gabriele P, Mauro F, Menegotti L, Monti $\mathrm{AF}$, Stasi $\mathrm{M}$ and Fiorino $\mathrm{C}$ : Increasing the risk of late rectal bleeding after high dose radiotherapy for prostate cancer: the case of previous abdominal surgery. Results from a prospective trial. Radiother Oncol 103: 252-255, 2012.

28 Denham JW and Hauer-Jensen M: The radiotherapeutic injurya complex 'wound'. Radiother Oncol 63: 129-145, 2002.

29 Fuccio L, Frazzoni L and Guido A: Prevention of pelvic radiation disease. World J Gastrointest Pharmacol Ther 6: 1-9, 2015.

30 Fuccio L, Guido A, Laterza L, Eusebi LH, Busutti L, Bunkheila F, Barbieri E and Bazzoli F: Randomised clinical trial: preventive treatment with topical rectal beclomethasone dipropionate reduces post-radiation risk of bleeding in patients irradiated for prostate cancer. Aliment Pharmacol Ther 34: 628637, 2011.

31 Ippolito E, Deodato F, Macchia G, Massaccesi M, Digesu' C, Pirozzi GA, Spera G, Marangi S, Annoscia E, Cilla S, Piermattei A, Valentini V, Cellini N, Ingrosso M and Morganti AG: Early radiation-induced mucosal changes evaluated by proctoscopy: Predictive role of dosimetric parameters. Radiother Oncol 104: 103-108, 2012.

32 Fellin G, Rancati T, Fiorino C, Vavassori V, Antognoni P, Baccolini M, Bianchi C, Cagna E, Borca VC, Girelli G, Iacopino B, Maliverni G, Mauro FA, Menegotti L, Monti AF, Romani F, Stasi M and Valdagni R: Long-term rectal function after highdose prostatecancer radiotherapy: Results from a prospective cohort study. Radiother Oncol 110: 272-277, 2014.

33 Miralbell R, Mollà M, Rouzaud M, Hidalgo A, Toscas JI, Lozano J, Sanz S, Ares C, Jorcano S, Linero D and Escudè L: Hypofractionated boost to the dominant tumor region with intensity-modulated stereotactic radiotherapy for prostate cancer: a sequential dose escalation pilot study. Int J Radiat Oncol Biol Phys 78: 50-57, 2010. 